\title{
Immunocytochemistry Test of Protein BRAF Expression for Diagnosis of Well Differentiated Thyroid Carcinoma
}

\author{
I. Wayan Sudarsa Elvis Deddy Kurniawan Pualillin \\ Putu Anda Tusta Adiputra Ida Bagus Tjakra Wibawa Manuaba
}

Surgical Oncology Division, Department of Surgery, Faculty of Medicine, Udayana

University, Sanglah General Hospital, Denpasar, Indonesia

\section{Keywords}

Immunocytochemistry test - BRAF expression - Thyroid carcinoma - FNAB

\begin{abstract}
Background: Thyroid carcinoma generally has a good prognosis. The main focus of current research on thyroid carcinoma is to increase the accuracy of preoperative diagnosis of thyroid nodules. When the result of fine needle aspiration biopsy (FNAB) is indeterminate, clinicians often have doubts in determining the surgical management. Objective: Protein BRAF expression analysis can help improve the accuracy of FNAB and optimize the management of differentiated thyroid carcinoma. Methods: This study is a diagnostic test performed from October 2016 at Sanglah General Hospital with 38 patients as subjects who fulfilled the inclusion criteria. Data is being presented in descriptive form before diagnostic test is done to determine sensitivity, specificity, positive predictive value, negative predictive value and the accuracy of immunocytochemistry test for BRAF on indeterminate thyroid nodule. Results: Thirty-eight samples met the inclusion criteria during the study period. Three samples were male $(7.9 \%)$
\end{abstract}


and 35 samples (92.1\%) were female. The mean age of the sample was 45.21 years (SD \pm 10.910 years) with ages ranging from 23 to 66 years. Of the 12 samples undergoing isthmolobectomy, 7 samples (58.4\%) were determined to be malignant from histopathological results. The sensitivity value of BRAF immunocytochemistry test is $45.45 \%$ with a specificity value of $81.25 \%$, a positive predictive value of $76.92 \%$, a negative predictive value of $52 \%$ and an accuracy of $60.50 \%$. Analysis of the receiver operator (ROC) curve shows the area under the curve (AUC) of $63.4 \%$ with a confidence interval of $45.5-81.2 \%$. Conclusion: Immunocytochemistry BRAF test have a reliable diagnostic value and can be taken into consideration in the preoperative diagnosis of thyroid malignancies.

(C) 2018 The Author(s)

Published by S. Karger AG, Basel

\section{Introduction}

Thyroid carcinoma generally has a good prognosis. The main focus of current research on thyroid carcinoma is how to improve the accuracy of preoperative diagnosis of thyroid nodules. The aim of these efforts is to improve the efficiency of differentiated thyroid carcinoma management. Fine needle aspiration biopsy (FNAB) is a conventional technique for identifying malignant thyroid nodules before surgery and is very effective. Although FNAB examination can differentiate between benign and malignant nodules, there are still 10-15\% of cases which cannot be inferred and named indeterminate nodules (Bethesda C3-C5), where cytology tests cannot distinguish between benign and malignant neoplasms [1].

Thyroid cancer originating from follicular thyroid cells is the most common thyroid malignancies and accounts for $95 \%$ of cases [2]. This malignancy is further divided into well differentiated thyroid cancer (DTC), poorly differentiated thyroid cancer (PDTC), anaplastic thyroid cancer (ATC). The 4 most common mutations related to DTC are: point mutation from BRAF, RAS rearrangements, RET/PTC, and Pax8/PPAR $\gamma$ [3].

If the accuracy of the FNAB can be increased by adding a BRAF analysis, it will help in identifying the nodule. This study aims to systematically review the accuracy of the cytology determination test of BRAFV600E mutation status in addition to conventional FNAB in patients with suspicious thyroid nodules.

\section{Materials and Methods}

This research is a diagnostic value of immunocytochemistry BRAF test on indeterminant thyroid nodule of Oncologic Surgery Division Faculty of Medicine Udayana University-Sanglah General Hospital. All patient with thyroid nodules who met the inclusion and exclusion criteria, underwent ultrasonography (USG) of the neck and thyroid function tests (FT4 and TSH). Patient suitable for surgical management are consulted to the anesthesiologist, as well as internist and cardiologist. FNAB is done according to the procedure and two sample slides are obtained.

The slide will be sent for the BRAF immunocytochemistry test using the BRAF antibody kit from Genetex (GTX 84812). The maximum value is determined using an H-score, where if the data distribution is normal, the average $\mathrm{H}$ score will be used as the maximum value and if 
the data is not normally distributed, the median H-score is used. The sample will be divided into 2 groups, BRAF positive and BRAF negative. The data is analyzed using $2 \times 2$ table with sensitivity, specificity, positive predictive value, negative predictive value and accuracy as the outcome.

\section{Results}

There were 38 research samples that met the inclusion criteria during the mentioned period. As many as 3 samples (7.9\%) were male and 35 samples (92.1\%) were female. The mean age of the sample was 45.21 years ( $S D \pm 10.910$ years) with ages ranging from 23 to 66 years. From 38 samples with follicular neoplasms, most of the samples ( 16 samples, $42.1 \%$ ) revealed benign histopathological results but only 26 samples (68.4\%) underwent total thyroidectomy (Table 1).

From 12 samples that underwent isthmolobectomy, 7 of the samples (58.4\%) were malignant. As shown in Table 2, there were 22 samples (42.1\%) with malignant histopathological results and 16 (57.9\%) were benign. For immunocytochemistry BRAF tests, the true positive value is 10 , and the false positive value is 3 . While the true negative value is 13 and the false negative value is 12 .

According to the results, the sensitivity value of the BRAF test can be calculated to be $45.45 \%$, specificity value to $81.25 \%$, positive predictive value to $76.92 \%$, negative predictive value to $52.00 \%$ and accuracy to $60.50 \%$. Analysis of receiver operator curve (ROC) shows that the area under the curve (AUC) is $63.4 \%$ with a confidence interval of $45.5-81.2 \%$ (Fig. 1).

\section{Discussion}

This study was conducted to evaluate the sensitivity, specificity, positive predictive value (PPV), negative predictive value (NPV), and immunocytochemistry accuracy of BRAF test in the diagnosis of thyroid nodules with cytology of follicular neoplasms. The number of samples collected was 38 and all samples underwent immunocytochemistry BRAF test preoperatively. The result of the immunocytochemistry compared to the gold standard histopathology test. From the 38 samples, 3 (7.9\%) were male and 35 (92.1\%) were women with an average age of 45.21 years ( $S D \pm 10.91$ years). This figure is similar to other studies stating that the majority of patients with thyroid cancer are female in the older age group $[4,5]$.

\section{Preoperative Immunocytochemistry BRAF Test on Thyroid Nodule}

The specificity, sensitivity, PPV, NPV, and accuracy of preoperative immunocytochemistry BRAF tests were $45.45,81.25,76.92,52.00$, and $60.50 \%$ respectively. Analysis of the receiver operator curve (ROC) shows the area under the curve (AUC) is $63.4 \%$ with a confidence interval (CI) of 45.5-81.2\%. From the 38 samples with cytology results of follicular neoplasms, there were 22 samples with malignant histopathological results and 26 samples underwent total thyroidectomy. Seven of the 22 samples with malignant histopathological results underwent isthmolobectomy. From the sample, we did not find any histopathological results 
consisting of thyroiditis, follicular carcinoma, medullar carcinoma or hurthle cell carcinoma. We hope that the additional BRAF test on FNAB will be the standard test in the preoperative diagnosis of thyroid nodules.

Similar research was conducted by Wobker et al. in 2015, they performed immunocytochemistry tests on 46 samples with papillary carcinoma from the thyroid and received a sensitivity value of $63.6 \%$, a specificity value of 58.3\%, PPV 58.3\% and NPV 63.6\% [6]. The difference in results might be due to differences in criteria inclusion used. Wobker et al added malignancy samples with FNAB results into their study, in which 20 of the 46 samples were papillary thyroid carcinoma. Not only that, they did not perform FNAB before surgery, but tests were carried out on histopathological samples obtained from surgery.

The Difference between Immunocytochemistry BRAF Test with Molecular Diagnostic Test of Thyroid Nodules

Other studies related to the diagnosis of preoperative thyroid cancer have been carried out using the method of classifying the gene expression of 167 genes [7], the genetic marker panel using 8 molecular signals [3], and Galectin-3 [8]. A meta-analysis conducted on the diagnostic value of BRAF V600E mutation analysis using FNAB also showed a relatively low sensitivity value $(44.2 \%)$. This study concludes that the role of BRAF analysis through preoperative FNAB is determined by the status of the prevalence of BRAF mutations in subcategories of indeterminant thyroid nodules [9].

Our research showed that the immunocytochemistry BRAF test has a PPV of $76.92 \%$ with NPV of $52.00 \%$ and generally has the potential to serve as "Rule in" preoperative diagnostic test for thyroid nodule. This result is in accordance with other research that stated the presence of single gene point mutation on BRAF [10]. The result of the immunocytochemistry BRAF test is different with the Afirma GECC test which is a "Rule out" diagnostic test on thyroid nodule (PPV immunocytochemistry BRAF vs. PPV Afirma GEC are 76.92 vs. 37\%; NPV immunocytochemistry BRAF vs. NPV Afrrma GEC are 52.00 vs. 95\%) [7]. Similar comparison is also made by comparing immunocytochemistry BRAF test with ThyroSeq (PPV immunocytochemistry BRAF vs. PPV ThyroSeq are 79.92 vs. 87\%) [11].

\section{Strength and Weakness of Immunocytochemistry BRAF Test}

One of the strengths of our study is that we use tests preoperatively, so the clinical feasibility of the procedure can be performed. In addition, we tested the specific subpopulations of patients with follicular neoplasms. One of the difficulties encountered is the lack of previous trials using preoperative immunocytochemistry techniques in patients with follicular neoplasms.

We use the semiquantitative $\mathrm{H}$ score method to determine the overexpression of BRAF from the FNAB results. To date, the role of BRAF in the diagnosis of thyroid carcinoma is controversial. The weakness of our study is we did not receive other benign histopathology results apart from follicular adenomas and other types of malignant histopathological result besides papillary carcinoma and its variants. In addition, other factors such as lack of consensus regarding immunocytochemistry BRAF procedural testing in thyroid nodules, differences in BRAF antibodies used and the presence of lymphocytes in great amount on FNAB can affect outcomes $[5,9,12]$. 


\section{Conclusion}

Immunocytochemistry BRAF tests have a reliable diagnostic value and can be considered in the preoperative diagnosis of thyroid malignancy (AUC 63.4\%; CI 45.5-81.2\%). Further studies need to be carried out for a larger sample group to evaluate BRAF expression in benign thyroid lesions, follicular thyroid carcinoma (FTC), medullary thyroid carcinoma (MTC) and poor differentiation / anaplastic thyroid carcinoma (ATC).

\section{Statement of Ethics}

This research article has been approved by the Local Ethical Committee and all subjects who participate in this study have signed an ethical approval form.

\section{Disclosure Statement}

None.

\section{Funding Sources}

None.

\section{References}

1 Ali SZ. Thyroid cytopathology: bethesda and beyond. Acta Cytol. 2011;55(1):4-12.

2 Xing M. Molecular pathogenesis and mechanisms of thyroid cancer. Nat Rev Cancer. 2013 Mar;13(3):184-99.

3 Nikiforov YE, Nikiforova MN. Molecular genetics and diagnosis of thyroid cancer. Nat Rev Endocrinol. 2011 Aug;7(10):569-80.

4 Faquin WC. The thyroid gland: recurring problems in histologic and cytologic evaluation. Arch Pathol Lab Med. 2008 Apr;132(4):622-32.

5 Haugen BR. 2015 American Thyroid Association Management Guidelines for Adult Patients with Thyroid Nodules and Differentiated Thyroid Cancer: what is new and what has changed? Cancer. 2017 Feb;123(3):372-81.

6 Wobker SE, Kim LT, Hackman TG, Dodd LG. Use of BRAF v600e immunocytochemistry on FNA direct smears of papillary thyroid carcinoma. Cancer Cytopathol. 2015 Sep;123(9):531-9.

7 Alexander EK, Kennedy GC, Baloch ZW, Cibas ES, Chudova D, Diggans J, et al. Preoperative diagnosis of benign thyroid nodules with indeterminate cytology. N Engl J Med. 2012 Aug;367(8):705-15.

8 Kato MA, Fahey TJ 3rd. Molecular markers in thyroid cancer diagnostics. Surg Clin North Am. 2009 Oct;89(5):1139-55.

9 Su X, Jiang X, Xu X, Wang W, Teng X, Shao A, et al. Diagnostic value of BRAF (V600E)-mutation analysis in fine-needle aspiration of thyroid nodules: a meta-analysis. Onco Targets Ther. 2016 Apr;9:2495-509.

10 Bernet V, Hupart KH, Parangi S, Woeber KA. AACE/ACE disease state commentary: molecular diagnostic testing of thyroid nodules with indeterminate cytopathology. Endocrine practice : official journal of the American College of Endocrinology and the American Association of Clinical Endocrinologists. 2014;20:3603.

11 Nikiforov YE, Carty SE, Chiosea SI, Coyne C, Duvvuri U, Ferris RL, et al. Impact of the Multi-Gene ThyroSeq Next-Generation Sequencing Assay on Cancer Diagnosis in Thyroid Nodules with Atypia of Undetermined 
Significance/Follicular Lesion of Undetermined Significance Cytology. Thyroid : official journal of the American Thyroid Association. 2015;25:1217-23.

12 Nikiforova MN, Kimura ET, Gandhi M, Biddinger PW, Knauf JA, Basolo F, et al. BRAF mutations in thyroid tumors are restricted to papillary carcinomas and anaplastic or poorly differentiated carcinomas arising from papillary carcinomas. J Clin Endocrinol Metab. 2003 Nov;88(11):5399-404.

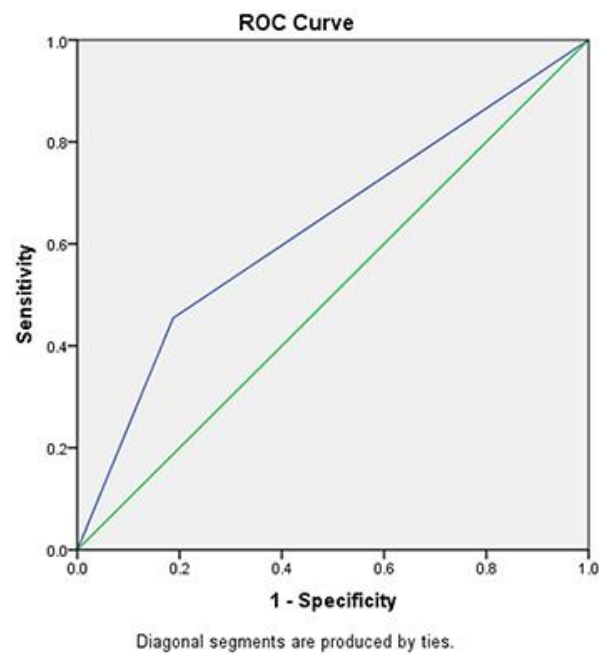

Fig. 1. Area under the curve of immunocytochemistry BRAF test.

Table 1. Characteristics of research sample

\begin{tabular}{llc}
\hline Variables & & $n(\%)$ \\
\hline Sex & female & $35(92.1)$ \\
& male & $3(7.9)$ \\
Age groups & $\leq 40$ years old & $14(36.8)$ \\
& $>40$ years old & $24(63.2)$ \\
Therapy & isthmolobectomy & $12(31.6)$ \\
& total thyroidectomy & $26(68.4)$ \\
FNAB & neoplasm follicular (Bethesda IV) & $37(97.37)$ \\
& suspect malignancy (Bethesda V) & $1(2.63)$ \\
Histopathology & struma adenomatous/follicular Adenoma & $16(42.1)$
\end{tabular}




\section{Case Reports in Oncology}

Table 2. The relationship between immunocytochemistry BRAF diagnostic test with histopathology results

\begin{tabular}{lllll}
\hline & \multicolumn{2}{l}{ Histopathology } & \multirow{2}{*}{ Total } \\
\cline { 3 - 4 } & & malignant & benign & \\
\hline Immunocytochemistry BRAF & positive & 10 & 3 & 13 \\
& negative & 12 & 13 & 25 \\
\hline Total & & 22 & 16 & 38 \\
\hline
\end{tabular}

Sudarsa et al.: Immunocytochemistry Test of Protein BRAF Expression for Diagnosis of

Well Differentiated Thyroid Carcinoma

(c) 2018 The Author(s). Published by S. Karger AG, Basel www.karger.com/cro 